\title{
COMPORTAMENTOS RELACIONADOS À SAÚDE EM IDOSOS BRASILEIROS RESIDENTES NA COMUNIDADE: DADOS DO FIBRA - POLO UNICAMP
}

Thaila Maki Hiraga ${ }^{1}$

Samila Sathler Tavares Batistoni²

Anita Liberalesso $\mathrm{Neri}^{3}$

resumo

Buscou-se descrever e identificar associações entre comportamentos relacionados à saúde entre si e com variáveis sociodemográficas em idosos residentes na comunidade. O estudo, de caráter descritivo, populacional e de corte transversal, é parte das análises do banco eletrônico do Estudo FIBRA - polo Unicamp, com amostra proba-

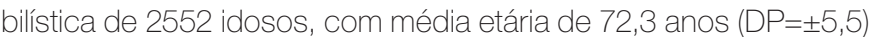
e composta predominantemente por mulheres (66\%). Análises de

1 Graduada em Fisioterapia. Especializada em Gerontologia na Universidade Federal de São Paulo. Mestre em Gerontologia pela Faculdade de Ciências Médicas da Universidade Estadual de Campinas (Unicamp). E-mail: thailamaki@yahoo.com.br.

2 Graduada em Psicologia. Doutora em Educação pela Universidade Estadual de Campinas. Professora titular de Graduação e Pós-Graduação em Gerontologia da Escola de Artes, Ciências e Humanidades da Universidade de São Paulo (USP). E-mail: samilabatistoni@gmail.com.

3 Graduada em Psicologia. Doutora em Psicologia pelo Instituto de Psicologia da USP. Coordenadora do Programa de Pós-graduação em Gerontologia da Faculdade de Ciências Médicas da Universidade Estadual de Campinas. Professora titular na Faculdade de Educação da Universidade Estadual de Campinas (Unicamp). E-mail: anitalbn@uol.com.br. 
Correspondência Múltipla mostraram associações entre as variáveis, apontando para a tendência de formação de três perfis comportamentais da amostra: 1) "Engajados em comportamentos não-saudáveis", 2) "Engajados em Comportamentos Saudáveis" e 3) "Indiferentes ou passivos frente à saúde". Ratificando a literatura, os comportamentos relacionados à saúde de idosos devem ser considerados de forma multidimensional ao gerar sinergia entre si. Tais informações contribuem para o delineamento de estratégias de serviços em saúde e bem-estar para a população idosa.

palavras-chave

Comportamentos saudáveis. Envelhecimento. Idosos. Promoção da saúde.

\section{Introdução}

As literaturas clínicas e de pesquisa utilizam-se de termos como "comportamento de saúde" (WHITLEY et al., 2014), "comportamento em saúde" (LIMA-COSTA et al., 2009), "comportamentos relacionados à saúde" (ZAITUNE et al., 2012), "comportamentos saudáveis" (JOMAR; ABREU; GRIEP, 2014) ou mesmo "estilo de vida" (VRDOLJAK et al., 2014) ao se referirem às ações que afetam a saúde de forma positiva ou negativa por meio dos quais os indivíduos protegem, mantêm e promovem o próprio estado de saúde (DESCRITORES..., 2015). Tais comportamentos considerados modificáveis e promotores da própria saúde são essenciais na avaliação dos níveis de bem-estar, longevidade e qualidade de vida das pessoas (CRAMM et al., 2014). O grande contingente de idosos nas sociedades atuais tem incentivado e gerado oportunidades de pesquisa para uma compreensão mais ampla da atuação dos fatores comportamentais e do investimento na saúde ao longo da vida e na determinação de trajetórias saudáveis ou patológicas de envelhecimento dos mesmos (ALDWIN; PARK; SPIRO III, 2007).

Sob uma perspectiva histórica do estudo sobre o processo de envelhecimento e os comportamentos na determinação da saúde e da doença é possível reconhecer certo paralelismo entre as literaturas advindas da Gerontologia Social, Epidemiologia e perspectivas clínicas. A evolução das abordagens que partiam de uma visão teórica de envelhecimento enquanto declínio inevitável e imutável, de práticas clínicas passivas diante dos acometimentos do 
envelhecimento e de estudos epidemiológicos focados apenas em indicadores de mortalidade e morbidade, alinham-se hoje ao paradigma life span em saúde. Tal paradigma pressupõe que o desenvolvimento acontece ao longo de toda a vida, está sujeito a uma infinidade de influências evolutivas, sociohistóricas e ontogenéticas, e se modifica também na velhice (ALDWIN; PARK; SPIRO III, 2007; NERI, 2006), estimulando assim as ações em promoção da saúde, prevenção e educação em saúde desde a mais tenra idade.

No Brasil, têm sido levantados alguns indicadores comportamentais importantes para a descrição e associação com as condições de saúde de idosos (IBGE, 2014; NERI et al., 2013; TEJADA et al., 2013). Dentre eles, destacam-se os indicadores de prática regular de exercícios físicos, de uso de tabaco e consumo de bebidas alcoólicas, de utilização de serviços médicos e odontológicos, e de imunização (COSTA; NERI, 2011; FRANCISCO et al., 2011; MIOTTO; BARCELLOS; VELTEN, 2012; MOURA, 2013; SENGER et al., 2011; VIANA; RODRIGUES; TAVARES, 2014; VRDOLJAK et al., 2014; WHITLEY et al., 2014). A maior parte dos estudos descreve os comportamentos relacionados à saúde e apontam para algumas associações com fatores sociodemográficos, porém de forma isolada, sem buscar uma compreensão de como os comportamentos geram sinergia entre si, em prol de um estilo de vida ativo e saudável ou sedentário e patológico (IBGE, 2015; LÄMMLE et al., 2014; PRUCHNO; WILSON-GENDERSON, 2012; VARGAS; BITTENCOURT; BARROSO, 2014). Frente à necessidade de identificar grupos vulneráveis de idosos através de comportamentos em saúde e suas associações, o presente estudo teve como objetivo descrever e identificar associações entre comportamentos relacionados à saúde entre si e as variáveis sociodemográficas em idosos residentes na comunidade.

\section{Método}

Trata-se de um estudo descritivo, populacional, de corte transversal, com análise de dados secundários do banco eletrônico do estudo FIBRA (Fragilidade em Idosos Brasileiros) - polo Unicamp coletados de uma amostra probabilística de idosos acima de 65 anos residentes em setores censitários da zona urbana de sete municípios no período de 2008 a 2009 (NERI et al., 2013). Foram analisadas variáveis sociodemográficas - sexo (masculino ou feminino), faixa etária (65 a 69,70 a 74,75 a 70 ou 80 anos ou mais), escolaridade (sem escolaridade formal, 1 a 4,5 a 8 ou 9 anos ou mais), renda familiar (menos que 1,0, 1,1 a 3,0, 3,1 a 5,0, 5,1 a 10,0 ou mais que 10,0 salários mínimos) e estado civil (casado, solteiro, viúvo ou divorciado); comportamentos relacionados à saúde - prática regular 
de exercício físico (fisicamente ativo ou sedentário), tabagismo (fumante, não-fumante ou ex-fumante), consumo de bebidas alcoólicas (abstêmios ou consumidores de bebidas alcóolicas) e sua frequência (moderado ou alto), consumo abusivo de bebidas alcóolicas (consumidores ou consumidores abusivos de bebidas alcoólicas) e sua frequência (nunca, ocasional ou frequente), utilização de serviço médico (sim ou não) e odontológico (sim ou não),imunização com vacina da influenza (sim ou não).

Foram considerados para a prática regular de exercício físico 16 itens do Minnesota Leisure Time Activity Questionnaire utilizado por Costa e Neri (2011), considerando as recomendações do American College of Sports Medicine - ACSM e American Heart Association - AHA (NELSON et al., 2007; HASKELL et al., 2007) de idosos ativos aqueles que acumularam pelo menos 30 minutos de exercício de intensidade moderada pelo menos cinco vezes na semana, em taxa de equivalentes metabólicos (MET), de 3 a 6 MET, ou 20 minutos de exercício de intensidade vigorosa ( $>6 \mathrm{MET}$ ) pelo menos três vezes semanalmente. O consumo abusivo de bebidas alcoólicas, de acordo com os critérios de Dawson e colaboradores (2005), foi considerado o consumo maior ou igual a cinco doses em uma única ocasião no mês. Os dados coletados foram consolidados e tratados estatisticamente pelo programa The Statistical Analysis System for Windows, versão 9.2. SAS Institute Inc, 2002-2008, Cary, NC, USA por meio de análises descritivas, para todas as variáveis, o Teste Qui-Quadrado ou Exato de Fisher com nível de significância $p<0,5$ para as associações entre as características sociodemográficas e os comportamentos relacionados à saúde e a Análise de Correspondência Múltipla para associações entre comportamentos em saúde entre si.

\section{Resultados}

A amostra contou com 2552 idosos, predominância no sexo feminino (66\%), com maior frequência etária na faixa entre 65 e 74 anos, cuja média alcançou 72,3 anos $(\mathrm{DP}= \pm 5,5)$. Cerca de $60 \%$ dos idosos entrevistados relataram renda familiar de até três salários mínimos (SM), com renda média para a amostra de 3,9 SM $(\mathrm{DP}= \pm 4,9)$. No que se refere à escolaridade, $49,1 \%$ tinham um a quatro anos de estudo e 50,5\% eram casados. Cerca de $60 \%$ dos idosos foram classificados como sedentários, 9,4\% são fumantes atuais, com média de tempo de consumo de 43,6 anos ( $\mathrm{DP}= \pm 16,3$ ). Entretanto, $35 \%$ mencionou ser ex-fumantes ao longo da vida. Em relação ao consumo de bebida alcoólica, aproximadamente $30 \%$ dos participantes afirmaram consumir, com maior 
percentual fazendo uso em frequência moderada $(23,2 \%)$, contudo $70 \%$ foram classificados como "abstêmios". O consumo abusivo de bebidas alcoólicas foi identificado em $4,5 \%$ dos participantes, dos quais $2,2 \%$ relataram abuso ocasional e 2,3\% abuso frequente. A procura por serviços médicos e odontológicos foram respectivamente encontrados em $91 \%$ e 35,5\% da amostra. Em média, o número de consultas médicas ao ano foi de $4,4( \pm 4,8)$, ao passo que a média de consultas odontológicas foi de $0,9( \pm 2,1)$. Cerca de $80 \%$ dos participantes do estudo foram imunizados com a vacina da influenza no último ano.

De acordo com a Tabela 1, o comportamento da prática regular de exercício físico apresentou associação significativa com a idade, cujos dados apontaram para a redução da prevalência de idosos ativos com o aumento da idade $(\mathrm{p}=0,010)$; com a escolaridade, em que a redução da prevalência de idosos ativos relaciona-se com a diminuição dos anos estudados $(p=0,003)$; entre homens $(p<0,001)$ e casados $(p=0,010)$. O tabagismo associou-se significativamente com a idade, com a redução da prevalência de indivíduos fumantes com o aumento da idade ( $p=0,009)$; com o sexo masculino $(\mathrm{p}<0,001)$ e indivíduos que relataram ser casados ou divorciados $(\mathrm{p}=0,007)$. A frequência do consumo de bebidas alcoólicas apresentou associações significativas com a idade, na medida em que a maiores frequências de consumo aumentaram com a diminuição da idade $(\mathrm{p}=0,007)$; a escolaridade, cujos dados apontaram para o aumento do consumo com o aumento dos anos estudados ( $\mathrm{p}<0,001)$; homens $(\mathrm{p}<0,001)$ e casados ( $\mathrm{p}<0,001)$. O consumo abusivo de bebidas alcóolicas e sua frequência associaram-se significativamente com a renda familiar e escolaridade, com consumo abusivo à medida que aumentam a renda $(\mathrm{p}=0,023)$ e os anos estudados $(p=0,041)$, entre os homens $(p<0,001)$. A utilização de serviço médico associou-se significativamente entre mulheres $(p<0,001)$, com a renda e a escolaridade, na medida em que houve maior utilização de serviço médico com o aumento da renda $(p=0,028)$ e dos anos estudados $(p=0,035)$. A utilização de serviço odontológico apresentou associação significativa com a renda e a escolaridade, na medida em que houve maior utilização de serviço com o aumento da renda $(p<0,001)$ e dos anos estudados $(p<0,001)$. A imunização com vacina da influenza associou-se significativamente com a idade, com a redução deste comportamento com o aumento da idade ( $\mathrm{p}=0,042)$; a escolaridade, na medida em que houve redução do mesmo com a redução dos anos estudados $(p=0,012)$; renda $(p=0,019)$ e entre mulheres $(p=0,012)$.

Para análise multivariada dos dados, utilizou-se a análise de Correspondência Múltipla (Tabela 2 e Figura 1), uma técnica de análise adequada para dados categóricos, que permite analisar graficamente as relações existentes através da redução de dimensionalidade do conjunto de dados. As principais 
associações foram entre alta frequência de consumo de bebidas alcoólicas, consumo abusivo de bebidas alcóolicas, frequência alta do consumo abusivo de bebidas alcoólicas, tabagismo e não utilização de serviço médico; a prática regular de exercício físico foi associada à utilização de serviço odontológico, a imunização com vacina da influenza, utilização de serviço médico, não tabagismo e não consumo abusivo de bebidas alcoólicas; e a não prática regular de exercício físico foi associada a não utilização de serviço odontológico, não imunização com vacina da influenza e não consumo de bebidas alcóolicas.

Tabela 1 - Frequência percentual de idosos conforme comportamentos relacionados à saúde considerando as variáveis sociodemográficas. FIBRA - polo Unicamp, Brasil, 2008-2009.

Parte l.

\begin{tabular}{|c|c|c|c|c|c|c|}
\hline & \multicolumn{2}{|c|}{$\begin{array}{l}\text { Prática Regular } \\
\text { de Exercício Físico }\end{array}$} & \multirow[t]{3}{*}{ Valor-p } & \multicolumn{2}{|c|}{ Tabagismo } & \multirow[t]{2}{*}{ Valor-p } \\
\hline & Sedentário & Ativo & & $\begin{array}{c}\text { Não } \\
\text { fumante }\end{array}$ & Fumante & \\
\hline \multicolumn{6}{|l|}{$\begin{array}{l}\text { Idade } \\
\text { (anos) }\end{array}$} & \\
\hline $65-69$ & $470(36,3)$ & $405(39,8)^{\star}$ & $0,010^{1 *}$ & $856(37,0)$ & $111(46,6)^{*}$ & $0,009^{1 *}$ \\
\hline $70-74$ & $394(30,5)$ & $328(32,2)$ & & $722(31,2)$ & $74(31,1)$ & \\
\hline $75-79$ & $256(19,8)$ & $192(18,8)$ & & $453(19,6)^{\star}$ & $33(13,9)$ & \\
\hline$\geq 80$ & $174(13,4)^{\star}$ & $94(9,2)$ & & $283(12,2)$ & $20(8,4)$ & \\
\hline \multicolumn{7}{|l|}{ Sexo } \\
\hline Masculino & $381(29,4)$ & $415(40,7)^{\star}$ & $<0,001^{1 *}$ & $762(32,9)$ & $111(46,6)^{\star}$ & $<0,001^{1 *}$ \\
\hline Feminino & $913(70,6)^{\star}$ & $604(59,3)$ & & $1552(67,1)^{\star}$ & $127(53,4)$ & \\
\hline \multicolumn{7}{|l|}{$\begin{array}{l}\text { Renda } \\
\text { familiar } \\
\text { (SM) }\end{array}$} \\
\hline$<1,0$ & $125(11,3)$ & $73(8,4)$ & $<0,001^{1 *}$ & $214(10,7)$ & $29(13,6)$ & 0,4211 \\
\hline $1,1-3,0$ & $570(51,5)^{\star}$ & $376(43,1)$ & & $956(48,0)$ & $108(50,7)$ & \\
\hline $3,1-5,0$ & $230(20,8)$ & $212(24,3)^{\star}$ & & $445(22,3)$ & $42(19,7)$ & \\
\hline $5,1-10,0$ & $133(12,0)$ & $131(15,0)$ & & $251(12,6)$ & $25(11,7)$ & \\
\hline$>10,0$ & $49(4,4)$ & $80(9,2)$ & & $127(6,4)$ & $9(4,3)$ & \\
\hline \multicolumn{7}{|c|}{$\begin{array}{l}\text { Escolari- } \\
\text { dade (anos) }\end{array}$} \\
\hline 0 & $265(20,5)^{\star}$ & $166(16,3)$ & $0,003^{1 *}$ & $453(19,6)$ & $52(21,9)$ & $0,473^{1}$ \\
\hline $0-4$ & $663(51,3)$ & $505(49,6)$ & & $1131(48,9)$ & $121(51,1)$ & \\
\hline
\end{tabular}




\begin{tabular}{|c|c|c|c|c|c|c|}
\hline & \multicolumn{2}{|c|}{$\begin{array}{l}\text { Prática Regular } \\
\text { de Exercício Físico }\end{array}$} & \multirow[t]{2}{*}{ Valor-p } & \multicolumn{2}{|c|}{ Tabagismo } & \multirow[t]{2}{*}{ Valor-p } \\
\hline & Sedentário & Ativo & & $\begin{array}{c}\text { Não } \\
\text { fumante }\end{array}$ & Fumante & \\
\hline $5-8$ & $220(17,0)$ & $190(18,7)$ & & $426(18,4)$ & $35(14,8)$ & \\
\hline$\geq 9$ & $145(11,2)$ & $157(15,4)^{\star}$ & & $303(13,1)$ & $29(12,2)$ & \\
\hline \multicolumn{7}{|c|}{ Estado civil } \\
\hline Casado & $621(48,1)$ & $552(54,2)^{*}$ & $0,010^{1 *}$ & $1157(50,1)$ & $130(54,8)^{\star}$ & $0,007^{1 *}$ \\
\hline Solteiro & $120(9,3)^{\star}$ & $69(6,8)$ & & $188(8,1)$ & $22(9,2)$ & \\
\hline Divorciado & $102(7,9)$ & $64(6,3)$ & & $162(7,0)$ & $26(11,0)$ & \\
\hline Viúvo & $448(34,7)$ & $333(32,7)$ & & $804(34,8)^{\star}$ & $59(25,0)$ & \\
\hline
\end{tabular}

1 Teste Qui-quadrado. ${ }^{2}$ Teste Exato de Fisher. ${ }^{*} p<0,05$.

Parte II.

\begin{tabular}{|c|c|c|c|c|c|c|c|}
\hline & \multicolumn{3}{|c|}{$\begin{array}{c}\text { Frequência do consumo } \\
\text { de álcool }\end{array}$} & \multirow[t]{2}{*}{ Valor-p } & \multicolumn{2}{|c|}{$\begin{array}{l}\text { Consumo Abusivo } \\
\text { de álcool }\end{array}$} & \multirow[t]{2}{*}{ Valor-p } \\
\hline & Nunca & Moderada & Alta & & Não & Sim & \\
\hline \multicolumn{8}{|l|}{$\begin{array}{l}\text { Idade } \\
\text { (anos) }\end{array}$} \\
\hline $65-69$ & \multirow{3}{*}{$\begin{array}{c}629(35,6) \\
561 \\
(31,7)^{\star} \\
355(20,1)\end{array}$} & \multirow{2}{*}{$\begin{array}{c}254 \\
(43,6)^{\star} \\
179(30,7)\end{array}$} & $67(40,4)^{\star}$ & \multirow[t]{3}{*}{$0,007^{1 *}$} & $885(37,2)$ & $52(46,9)$ & \multirow[t]{4}{*}{$0,116^{1}$} \\
\hline $70-74$ & & & $46(27,7)$ & & $744(31,3)$ & $35(31,5)$ & \\
\hline $75-79$ & & $97(16,6)$ & $28(16,9)$ & & $463(19,5)$ & $15(13,5)$ & \\
\hline$\geq 80$ & $222(12,6)$ & $53(9,1)$ & $25(15,0)$ & & $285(12,0)$ & $9(8,1)$ & \\
\hline
\end{tabular}

Sexo

\begin{tabular}{|c|c|c|c|c|c|c|}
\hline Masculino & $462(26,1)$ & $271(46,5)$ & $\begin{array}{c}123 \\
(741)^{*}\end{array}$ & $<0,001^{1 *}$ & $758(31,9)$ & $84(75,7)^{\star}$ \\
\hline Feminino & $\begin{array}{c}1305 \\
(73,9)^{\star}\end{array}$ & $\begin{array}{c}312 \\
(53,5)^{\star}\end{array}$ & $43(25,9)$ & & $\begin{array}{c}1619 \\
(68,1)^{\star}\end{array}$ & $27(24,3)$ \\
\hline
\end{tabular}

\section{Renda}

familiar

(SM)

\begin{tabular}{|c|c|c|c|c|c|c|c|}
\hline$<1,0$ & $\begin{array}{c}191 \\
(12,7)^{\star}\end{array}$ & $39(7,5)$ & $5(3,4)$ & $<0,0011^{1 *}$ & $223(10,9)$ & $6(6,1)$ & $0,023^{1 *}$ \\
\hline $1,1-3,0$ & $\begin{array}{c}791 \\
(52,4)^{\star}\end{array}$ & $205(39,5)$ & $53(36,6)$ & & $\begin{array}{c}996 \\
(48,6)^{\star}\end{array}$ & $40(41,3)$ & \\
\hline $3,1-5,0$ & $311(20,6)$ & $137(26,4)$ & $33(22,8)$ & & $450(21,9)$ & $28(28,9)^{*}$ & \\
\hline $5,1-10,0$ & $154(10,2)$ & $183(16,0)$ & $36(24,8)^{\star}$ & & $261(12,7)$ & $11(11,3)$ & \\
\hline$>10,0$ & $62(4,1)$ & $55(10,6)$ & $18(12,4)^{\star}$ & & $121(5,9)$ & $12(12,4)^{\star}$ & \\
\hline
\end{tabular}

Continua 


\begin{tabular}{|c|c|c|c|c|c|c|c|}
\hline & \multicolumn{3}{|c|}{$\begin{array}{l}\text { Frequência do consumo } \\
\text { de álcool }\end{array}$} & \multirow[t]{2}{*}{ Valor-p } & \multicolumn{2}{|c|}{$\begin{array}{l}\text { Consumo Abusivo } \\
\text { de álcool }\end{array}$} & \multirow[t]{2}{*}{ Valor-p } \\
\hline & Nunca & Moderada & Alta & & Não & Sim & \\
\hline \multicolumn{8}{|l|}{$\begin{array}{l}\text { Escola- } \\
\text { ridade } \\
\text { (anos) }\end{array}$} \\
\hline 0 & $\begin{array}{c}412 \\
(23,3)^{\star}\end{array}$ & $58(10,0)$ & $19(11,4)$ & $<0,001^{1 *}$ & $\begin{array}{c}471 \\
(19,8)^{\star}\end{array}$ & $14(12,6)$ & $0,041^{1 *}$ \\
\hline $0-4$ & $878(49,8)$ & $278(47,7)$ & $84(50,6)$ & & $\begin{array}{l}1179 \\
(49,6)\end{array}$ & $50(45,1)$ & \\
\hline $5-8$ & $297(16,8)$ & $132(22,6)$ & $27(16,3)$ & & $423(17,8)$ & $26(23,4)^{*}$ & \\
\hline$\geq 9$ & $178(10,1)$ & $\begin{array}{c}115 \\
(197)^{\star}\end{array}$ & $36(21,7)^{\star}$ & & $302(12,7)$ & $21(18,9)^{\star}$ & \\
\hline \multicolumn{8}{|l|}{$\begin{array}{l}\text { Estado } \\
\text { civil }\end{array}$} \\
\hline Casado & $823(46,7)$ & $\begin{array}{c}335 \\
(57,6)^{\star}\end{array}$ & $\begin{array}{c}110 \\
(66,3)^{\star}\end{array}$ & $<0,001^{1 *}$ & $\begin{array}{l}1180 \\
(49,7)\end{array}$ & $69(62,2)$ & 0,0521 \\
\hline Solteiro & $159(9,0)$ & $43(7,4)$ & $4(2,4)$ & & $199(8,4)$ & $7(6,3)$ & \\
\hline Divorciado & $131(7,4)$ & $35(6,0)$ & $19(11,4)$ & & $175(7,4)$ & $9(8,1)$ & \\
\hline Viúvo & $\begin{array}{c}651 \\
(36,9)^{\star}\end{array}$ & $\begin{array}{c}169 \\
(29,0)^{\star}\end{array}$ & $33(19,9)$ & & $819(34,5)$ & $26(23,4)$ & \\
\hline
\end{tabular}

1 Teste Qui-quadrado. ${ }^{2}$ Teste Exato de Fisher. ${ }^{*} \mathrm{p}<0,05$.

Parte III.

\begin{tabular}{|c|c|c|c|c|c|c|c|}
\hline & \multicolumn{3}{|c|}{ Frequência do abuso de álcool } & \multirow[t]{2}{*}{ Valor-p } & \multicolumn{2}{|c|}{$\begin{array}{l}\text { Utilização de serviço } \\
\text { médico }\end{array}$} & \multirow[t]{2}{*}{ Valor-p } \\
\hline & Nunca & Ocasional & Alta & & Não & Sim & \\
\hline \multicolumn{8}{|l|}{$\begin{array}{l}\text { Idade } \\
\text { (anos) }\end{array}$} \\
\hline $65-69$ & $\begin{array}{c}885 \\
(37,2)\end{array}$ & $27(49,1)$ & $25(44,6)$ & $0,245^{1}$ & $97(43,1)$ & $833(37,6)$ & $0,112^{1}$ \\
\hline 70-74 & $\begin{array}{l}744 \\
(31,3)\end{array}$ & $17(30,9)$ & $18(32,1)$ & & $70(31,1)$ & $698(31,6)$ & \\
\hline $75-79$ & $\begin{array}{c}463 \\
(19,5)\end{array}$ & $5(9,1)$ & $10(17,9)$ & & $30(13,3)$ & $432(19,5)$ & \\
\hline$\geq 80$ & $\begin{array}{l}285 \\
(12,0)\end{array}$ & $6(10,9)$ & $3(5,4)$ & & $28(12,5)$ & $249(11,3)$ & \\
\hline
\end{tabular}

Sexo

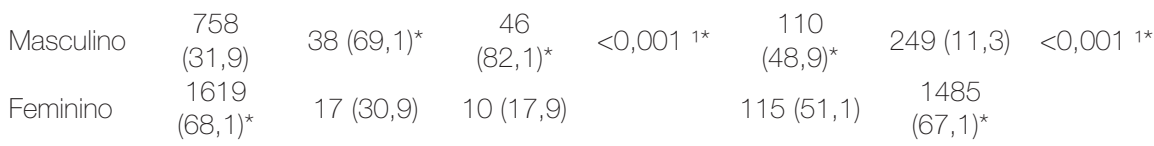
Continua 


\begin{tabular}{|c|c|c|c|c|c|c|c|}
\hline & \multicolumn{3}{|c|}{ Frequência do abuso de álcool } & \multirow[t]{2}{*}{ Valor-p } & \multicolumn{2}{|c|}{$\begin{array}{l}\text { Utilização de serviço } \\
\text { médico }\end{array}$} & \multirow[t]{2}{*}{ Valor-p } \\
\hline & Nunca & Ocasional & Alta & & Não & Sim & \\
\hline \multicolumn{8}{|l|}{$\begin{array}{l}\text { Renda } \\
\text { familiar } \\
\text { (SM) }\end{array}$} \\
\hline$<1,0$ & $\begin{array}{c}223 \\
(10,9)^{\star}\end{array}$ & $5(10,4)^{\star}$ & $1(2,1)$ & $0,0266^{1 *}$ & $23(11,7)$ & $206(10,7)$ & $0,028^{1 *}$ \\
\hline $1,1-3,0$ & $\begin{array}{c}996 \\
(48,6)^{\star}\end{array}$ & $17(35,4)$ & $\begin{array}{c}23 \\
(46,9)^{\star}\end{array}$ & & $\begin{array}{c}103 \\
(52,5)^{*}\end{array}$ & $919(47,9)$ & \\
\hline $3,1-5,0$ & $\begin{array}{c}450 \\
(21,9)\end{array}$ & $17(35,4)^{\star}$ & $11(22,5)$ & & $47(24,0)$ & $423(22,0)$ & \\
\hline $5,1-10,0$ & $\begin{array}{c}261 \\
(12,7)\end{array}$ & $3(6,3)$ & $8(16,3)^{\star}$ & & $10(5,1)$ & $\begin{array}{c}255 \\
(12,3)^{*}\end{array}$ & \\
\hline$>10,0$ & $121(5,9)$ & $6(12,5)^{\star}$ & $6(12,2)^{\star}$ & & $13(6,7)$ & $116(6,1)$ & \\
\hline \multicolumn{8}{|l|}{$\begin{array}{l}\text { Escola- } \\
\text { ridade } \\
\text { (anos) }\end{array}$} \\
\hline 0 & $\begin{array}{c}471 \\
(19,8)\end{array}$ & $6(10,9)$ & $8(14,3)$ & $0,100^{1}$ & $57(25,3)^{\star}$ & $425(19,2)$ & $0,035^{1 *}$ \\
\hline $0-4$ & $\begin{array}{l}1179 \\
(49,6)\end{array}$ & $23(41,8)$ & $27(48,2)$ & & $108(48,0)$ & $\begin{array}{c}1079 \\
(48,8)^{\star}\end{array}$ & \\
\hline $5-8$ & $\begin{array}{l}423 \\
(17,8)\end{array}$ & $16(29,1)$ & $10(17,9)$ & & $42(18,7)$ & $408(18,5)$ & \\
\hline$\geq 9$ & $\begin{array}{c}302 \\
(12,8)\end{array}$ & $10(18.2)$ & $11(19,6)$ & & $18(8,0)$ & $\begin{array}{c}298 \\
(13,5)^{\star}\end{array}$ & \\
\hline \multicolumn{8}{|l|}{$\begin{array}{l}\text { Estado } \\
\text { civil }\end{array}$} \\
\hline Casado & $\begin{array}{r}1180 \\
(49,7)\end{array}$ & $35(63,6)$ & $34(60,7)$ & $0,074^{2}$ & $123(54,9)$ & $\begin{array}{r}1116 \\
(50,5)\end{array}$ & 0,3191 \\
\hline Solteiro & $199(8,4)$ & $5(9,2)$ & $2(3,6)$ & & $22(9,8)$ & $179(8,1)$ & \\
\hline Divorciado & $175(7,4)$ & $2(3,6)$ & $2(12,5)$ & & $13(5,8)$ & $168(7,6)$ & \\
\hline Viúvo & $\begin{array}{c}819 \\
(34,5)\end{array}$ & $13(23,6)$ & $13(23,2)$ & & $66(29,5)$ & $746(33,8)$ & \\
\hline
\end{tabular}

1 Teste Qui-quadrado. ${ }^{2}$ Teste Exato de Fisher. * $p<0,05$.

Parte IV.

\begin{tabular}{|c|c|c|c|c|c|c|}
\hline & \multicolumn{2}{|c|}{$\begin{array}{c}\text { Utilização de serviço } \\
\text { odontológico }\end{array}$} & \multirow[t]{2}{*}{ Valor-p } & \multicolumn{2}{|c|}{ Imunização contra gripe } & \multirow[t]{2}{*}{ Valor-p } \\
\hline & Não & Sim & & Não & Sim & \\
\hline \multicolumn{7}{|l|}{$\begin{array}{l}\text { Idade } \\
\text { (anos) }\end{array}$} \\
\hline $65-69$ & $594(36,5)$ & $363(40,6)$ & $0,143^{1}$ & $747(37,0)$ & $216(41,1)^{\star}$ & $0,042^{1 *}$ \\
\hline $70-74$ & $511(31,4)$ & $273(30,5)$ & & $622(30,9)$ & $170(32,4)$ & \\
\hline
\end{tabular}

Continua 


\begin{tabular}{|c|c|c|c|c|c|c|}
\hline & \multicolumn{2}{|c|}{$\begin{array}{c}\text { Utilização de serviço } \\
\text { odontológico }\end{array}$} & \multirow[t]{2}{*}{ Valor-p } & \multicolumn{2}{|c|}{ Imunização contra gripe } & \multirow[t]{2}{*}{ Valor-p } \\
\hline & Não & Sim & & Não & Sim & \\
\hline $75-79$ & $315(19,4)$ & $167(18,7)$ & & $391(19,4)$ & $93(17,7)$ & \\
\hline$\geq 80$ & $205(12,7)$ & $92(10,2)$ & & $256(12,7)^{\star}$ & $46(8,7)$ & \\
\hline \multicolumn{7}{|l|}{ Sexo } \\
\hline Masculino & $555(34,1)$ & $302(33,7)$ & $0,835^{1}$ & $713(35,4)^{\star}$ & $155(29,5)$ & $0,012^{1 *}$ \\
\hline Feminino & $1070(65,9)$ & $593(55,3)$ & & $1303(64,6)$ & $370(70,5)^{\star}$ & \\
\hline \multicolumn{7}{|l|}{$\begin{array}{l}\text { Renda } \\
\text { familiar } \\
\text { (SM) }\end{array}$} \\
\hline$<1,0$ & $183(13,0)$ & $60(7,8)$ & $<0,0011^{1 *}$ & $197(11,2)$ & $45(10,3)$ & $0,019^{1 *}$ \\
\hline $1,1-3,0$ & $759(53,9)^{\star}$ & $290(37,7)$ & & $869(49,4)^{\star}$ & $189(43,3)$ & \\
\hline $3,1-5,0$ & $289(20,5)$ & $192(24,9)$ & & $373(21,2)$ & $113(25,9)^{\star}$ & \\
\hline $5,1-10,0$ & $127(9,1)$ & $145(18,8)^{\star}$ & & $223(12,7)$ & $53(12,1)$ & \\
\hline$>10,0$ & $49(3,5)$ & $83(10,8)^{\star}$ & & $96(5,5)$ & $37(8,4)^{\star}$ & \\
\hline \multicolumn{7}{|c|}{$\begin{array}{l}\text { Escolari- } \\
\text { dade (anos) }\end{array}$} \\
\hline 0 & $383(23,6)^{\star}$ & $121(13,5)$ & $<0,0011^{1 *}$ & $389(19,8)$ & $105(20,0)$ & $0,012^{1 *}$ \\
\hline $0-4$ & $822(50,6)$ & $409(45,7)$ & & $1014(50,3)^{\star}$ & $232(44,3)$ & \\
\hline $5-8$ & $285(17,5)$ & $174(19,5)$ & & $362(18,0)$ & $99(18,9)$ & \\
\hline$\geq 9$ & $135(8,3)$ & $190(21,3)^{\star}$ & & $240(11,9)$ & $88(16,8)^{\star}$ & \\
\hline \multicolumn{7}{|c|}{ Estado civil } \\
\hline Casado & $797(49,0)$ & $470(52,6)$ & $0,186^{1}$ & $1039(51,6)$ & $241(46,0)$ & $0,186^{1}$ \\
\hline Solteiro & $131(8,1)$ & $79(8,9)$ & & $169(8,4)$ & $41(7,8)$ & \\
\hline Divorciado & $122(7,5)$ & $63(7,1)$ & & $140(6,9)$ & $48(9,2)$ & \\
\hline Viúvo & $575(35,4)$ & $280(31,4)$ & & $665(33,1)$ & $194(37,0)$ & \\
\hline
\end{tabular}

${ }^{1}$ Teste Qui-quadrado, ${ }^{2}$ Teste Exato de Fisher. ${ }^{\star} p<0,05$.

Fonte: Tabela elaborada pelas autoras.

Tabela 2 - Coordenadas das variáveis para o gráfico da Análise de Correspondência Múltipla (n=2132). FIBRA - polo Unicamp, Brasil, 2008-2009.

\begin{tabular}{|c|c|c|}
\hline & DIM1 & DIM2 \\
\hline \multicolumn{3}{|c|}{ Prática regular de exercício físico } \\
\hline Sedentário & $-0,0722$ & 0,4536 \\
\hline Ativo & 0,0930 & $-0,5848$ \\
\hline
\end{tabular}




\begin{tabular}{l|c|c}
\hline \multicolumn{1}{l|}{} & DIM1 & DIM2 \\
\hline Tabagismo & $-0,0712$ & $-0,0850$ \\
\hline Não fumante & 0,7879 & 0,9402 \\
\hline Fumante & & \multicolumn{1}{l}{} \\
\hline Frequência do consumo de bebidas alcóolicas & $-0,3375$ & 0,2480 \\
\hline Nunca & 0,4209 & $-0,9141$ \\
\hline Frequência moderada & 2,0805 & 0,6202 \\
\hline Frequência alta & &
\end{tabular}

\section{Consumo abusivo de bebidas alcóolicas}

\begin{tabular}{l|c|c}
\hline Não & $-0,2107$ & 0,0014 \\
\hline Sim & 4,3351 & $-0,0292$ \\
\hline
\end{tabular}

Frequência do consumo abusivo de bebidas alcóolicas

\begin{tabular}{l|c|c}
\hline Abuso frequente & 4,6285 & 1,4785 \\
\hline Nunca & $-0,2107$ & 0,0014 \\
\hline Abuso ocasional & 4,0588 & $-1,4481$ \\
\hline
\end{tabular}

\section{Utilização de serviço médico}

\begin{tabular}{l|c|c}
\hline Não & 0,3532 & 1,3337 \\
\hline Sim & $-0,0323$ & $-0,1220$ \\
\hline \multicolumn{1}{l|}{ Utilização de serviço odontológico } & $-0,0005$ & 0,3785 \\
\hline Não & 0,0009 & $-0,6501$ \\
\hline Sim & $-0,0611$ & 0,7159 \\
\hline Imunização contra gripe & 0,0152 & $-0,1784$ \\
\hline Não & &
\end{tabular}

Inércia de dimensões 1 e 2= 35,6\%. DIM= dimensão.

Fonte: Tabela elaborada pelas autoras. 
Figura 1 - Análise de Correspondência Múltipla (n=2132). FIBRA - polo Unicamp, Brasil, 2008-2009.

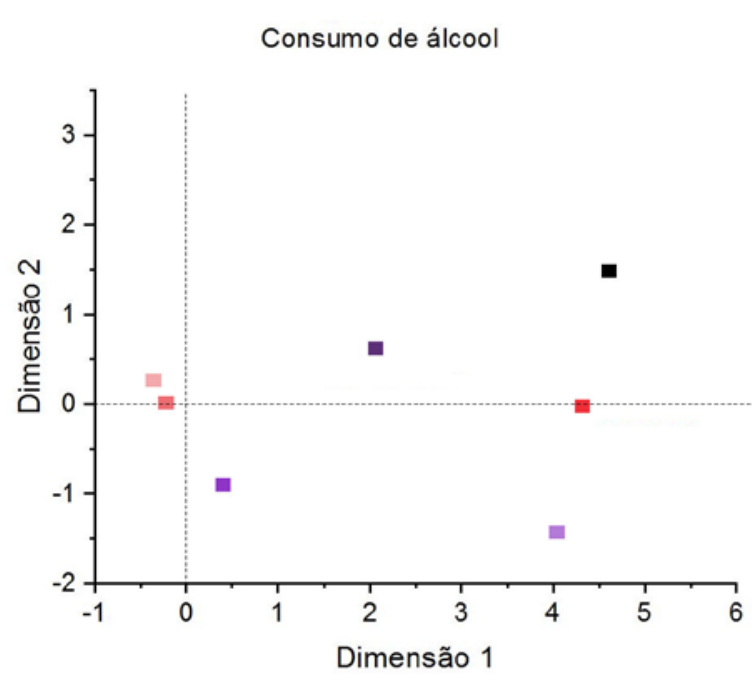

\section{Consumo de álcool}

- Frabus

- Freq Alta

- Freq Mod

- Freq Ocasion

11 Abstêmio

- N Abusa/Fr_Nunca

- Abusa

Uso do serviço médico e odontológico

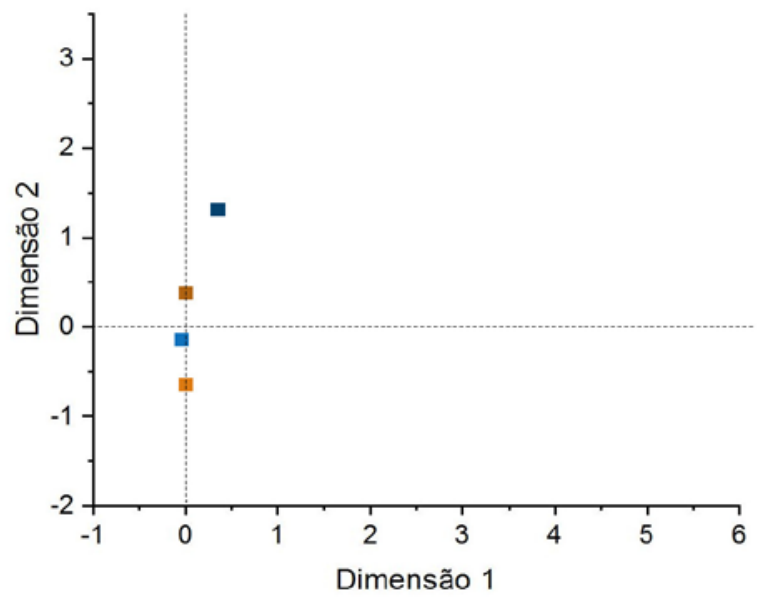

Uso serviço médico

- Não Méd

- Medic

Uso serviço odontol.

Não Odon

Odontol

Continua 
Relação entre Tabagismo e Exercícios Físicos

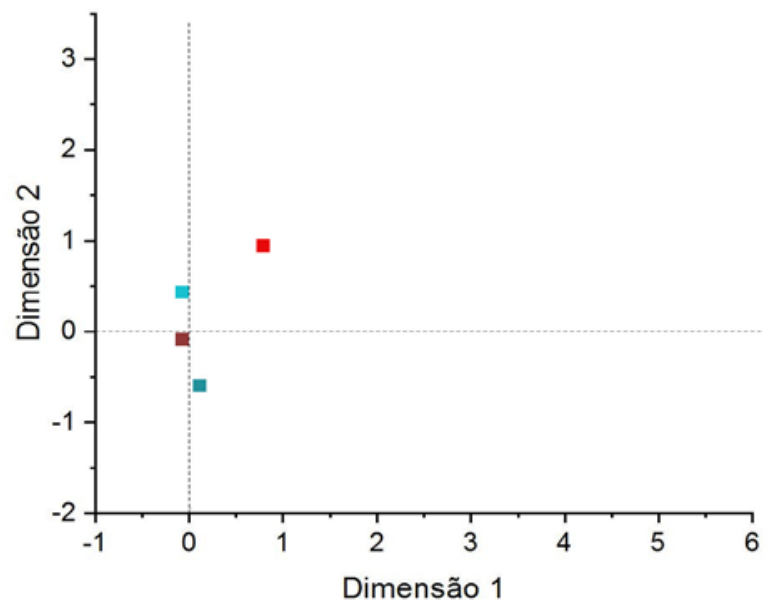

Vacina de gripe

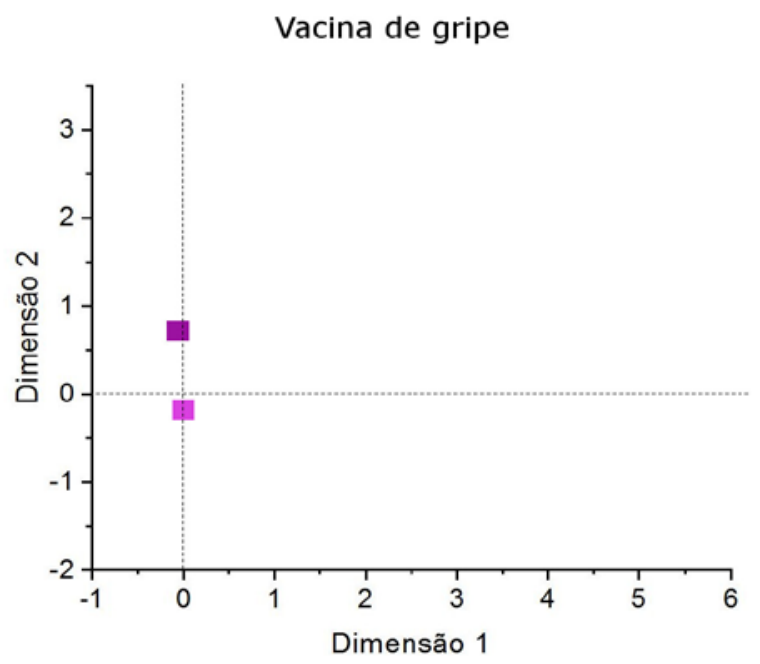

Tabagismo

- Tabag

- Não tabag

Exercícios Físicos

Sedent

Ativo
Vacina de gripe

Vacina

- Não vacin

A amostra para o presente estudo, como parte dos dados do estudo FIBRA - polo Unicamp, manteve a proporção de idosos, segundo as características sociodemográficas, semelhantes às já descritas em publicações anteriores (NERI 
et al., 2013) e com dados nacionais (BRASIL, 2014). É relevante a proporção de mulheres no estudo, dos idosos com baixa escolaridade e renda familiar.

No que se referiu à prática de exercício de físico, observou-se maior proporção de idosos sedentários, com percentual semelhante ao encontrado em outros estudos que também utilizaram o mesmo critério de classificação da ACSM e a AHA (COSTA; NERI, 2011; VRDOLJAK et al., 2014). A diversidade de critérios e nomenclaturas adotados dificulta as comparações entre os estudos sem antes verificar com atenção os parâmetros adotados. Prevalências inferiores do tabagismo foram encontradas em outros estudos (ZAITUNE et al., 2012; KENDIG et al., 2014), que, em parte, pode ser justificada pelo critério de inclusão de idosos na amostra a partir de 65 anos, retirando uma porcentagem significativa de possíveis fumantes, além da possibilidade de a maior inclusão de mulheres idosas advindas de cidades interioranas, apontando para efeitos de corte. A Pesquisa Nacional Brasileira sobre Álcool identificou valor semelhante ao consumo e valor menor ao abuso de álcool entre idosos do presente estudo (MADRUGA et al., 2010). Entretanto, pesquisas (BRASIL, 2014; VRDOLJAK et al., 2014) identificaram prevalência menor de consumidores de bebidas alcoólicas, principalmente por diferentes critérios adotados em relação a esse comportamento e as populações abordadas.

A procura por serviço médico é maior, resultado que corrobora com outros estudos (MIOTTO; BARCELLOS; VELTEN, 2012). Entretanto, em relação às consultas médicas anuais de idosos no Brasil, diferente prevalência foi encontrada, em torno de 16,5\% (IBGE, 2015). Quanto às consultas odontológicas, a prevalência semelhante à do nosso estudo foi apontada em pesquisa utilizando dados da ISACamp com $64,1 \%$ de idosos de Campinas sem consulta odontológica anual (FRANCISCO et al., 2011). A ausência de necessidade percebida tem sido citada como uma barreira para a utilização de serviços odontológicos, já que para muitos os problemas bucais não ameaçam à vida e os impactos no bem-estar não são óbvios (MIOTTO; BARCELLOS; VELTEN, 2012). É necessário olhar para as iniquidades em saúde a qual está diretamente relacionada à renda e escolaridade, e também se atentar às percepções individuais sobre os serviços (SMITH; GLAZIER; SIBLEY, 2010). Em relação à imunização com vacina da influenza, dados do estudo FIBRA foram coletados em meados do ano 2008 e 2009, e pesquisas brasileiras desenvolvidas com idosos nesse mesmo período encontraram prevalências mais baixas (FRANCISCO et al., 2011). A cobertura vacinal nacional da influenza correspondeu a 75\% em 2008 e 83,1\% em 2009 (BRASIL, 2009, 2010), o valor encontrado no estudo foi correspondente a esse intervalo. 
Em relação à idade, a prática regular de exercício físico, o tabagismo, a frequência moderada de consumo de álcool e a imunização com vacina da influenza mostraram associações. A prática regular de exercício associou-se a idosos mais jovens, assim como outros estudos (WHITLEY et al., 2014; VAGETTI et al., 2013). A progressão da idade parece associar-se à maior incapacidade funcional, maior prevalência de doenças crônicas, efeitos deletérios do próprio processo de envelhecimento e estereótipos negativos sobre o mesmo pelos idosos e pela sociedade (EMILE et al., 2014). Estudos apontam que os idosos que sobrevivem às taxas excessivas das mortes prematuras provocadas pelo tabagismo geram dados subestimados sobre o comportamento. Individualmente, o próprio idoso considera-se relativamente imune aos prejuízos causados pelo tabaco ou adquirindo experiências negativas são encorajados à cessação do uso (TEJADA et al., 2013).

Em relação ao consumo de álcool o Relatório de Status Global sobre Álcool e Saúde em grupos etários indica que, apesar de geralmente sofrer um declínio com o avanço da idade, indivíduos mais velhos consomem bebidas alcoólicas com maior frequência (OMS, 2014). Em relação à imunização, a progressão da idade associa-se com maior prevalência de incapacidade funcional, principalmente nos maiores de 80 anos e maior dificuldade de mobilidade até locais de vacinação e menor cobertura vacinal (PEREIRA et al., 2012). Da mesma forma, idosos mais jovens com melhor percepção de saúde podem desconsiderar a importância da imunização, influenciando a uma menor cobertura vacinal (CAMPOS et al., 2012).

Em relação ao sexo, a prática regular de exercício físico, tabagismo, consumo abusivo de álcool, utilização de serviço médico e imunização com vacina da influenza associaram-se significativamente. Homens são mais incentivados à realização de atividade física desde a infância e permanecem ativos até idades avançadas quando comparados às mulheres (LEBRÃO; LAURENTI, 2005). Dados históricos afirmam que homens fumam mais (VIANA; RODRIGUES; TAVARES, 2014). Homens bebem com maior frequência e em maior quantidade em relação às mulheres, justificado pela maior percentagem de indivíduos do sexo masculino com doenças relacionadas ao consumo (VARGAS; BITTENCOURT; BARROSO, 2014). Mulheres utilizam mais serviços médicos e aderem à imunização com vacina da influenza (PALACIOS-CENÃ et al., 2013), preocupam-se mais com a saúde, tem maior número de doenças crônicas, e consequentemente percebem potenciais riscos à saúde e utilizam-se dos serviços como exames preventivos e consultas periódicas com mais frequência que os indivíduos do sexo oposto (BRASIL, 2012). 
Em relação ao estado civil, os comportamentos prática regular de exercícios físicos, tabagismo, frequência de consumo de bebidas alcoólicas e imunização com vacina da influenza associaram-se significativamente. Indivíduos casados estiveram entre os mais ativos fisicamente (DUARTE et al., 2008). Idosos casados parecem consumir com maior frequência bebidas alcoólicas, o cônjuge pode influenciar negativamente a companheira e vice-versa, e os indivíduos que bebem são mais vulneráveis a danos sociais como problemas de relacionamento entre familiares e até morte (OMS, 2014). Além disso, o uso de tabaco pode ser considerado um fator negativo ou positivo, uma vez que aquele que fuma pode desencorajar o outro a abandonar o hábito, ou na medida que o companheiro que não fuma estimula o parceiro a cessar seu vício (SENGER et al., 2011; VIANA; RODRIGUES; TAVARES, 2014).

Em relação à escolaridade, a prática regular de exercício físico, o consumo, o consumo abusivo de bebidas alcoólicas e sua frequência, a utilização de serviço médico e odontológico, e a imunização com vacina da influenza associaram-se de forma significativa. A renda associou-se apenas com o consumo abusivo e sua frequência, a utilização de serviço médico e odontológico, e a imunização contra a gripe. A oportunidade da educação formal é um fator determinante sobre as condições de saúde (JOMAR; ABREU; GRIEP, 2014). Idosos com maior grau de instrução e renda têm mais acesso à informação e condições de vida que propiciam a incorporação de comportamentos mais saudáveis (VAGETTI et al., 2013; LOUVISON et al., 2008).

Considerando que os comportamentos tendem a atuar de forma associada ou mesmo sinérgica aumentando ou diminuindo a probabilidade de efeitos negativos ou positivos à saúde (DODD et al., 2010), pode-se identificar três possíveis perfis comportamentais na amostra: a) "Engajados em comportamentos não-saudáveis", uma vez que foi composto, em maioria, por indivíduos com alta frequência de consumo de bebidas alcoólicas, maior frequência de consumo abusivo de álcool, uso de tabaco e não utilização de serviço médico no último ano; b) "Engajados em Comportamentos Saudáveis", pois incluiu indivíduos com maior envolvimento com a prática regular de exercício físico, a utilização de serviço odontológico e médico no último ano, imunização com vacina da influenza, não tabagistas e não consumidores abusivos de bebidas alcoólicas; c) "Indiferentes ou passivos frente à saúde" uma vez composto, em maioria, por indivíduos que não praticam exercícios físicos regulares, não utilizaram serviço odontológico no último ano, não imunizados contra a gripe e que não consomem bebidas alcoólicas. Entre o perfil "Engajados em comportamentos não-saudáveis" e "Engajados em Comportamentos Saudáveis" muitos fatores poderiam justificar tais associações, já que ambos os perfis são 
complementares e opostos. O terceiro perfil "Indiferentes ou passivos frente à saúde" indicaria uma tendência daqueles indivíduos com uma postura de não agência à sua própria saúde, que apresentam comportamentos ora positivos ora negativos à saúde que se auto anulam, sem potencializar como nos outros perfis os benefícios e os prejuízos à saúde do idoso.

Em linhas gerais, considera-se que o presente estudo alcançou os objetivos traçados. Contudo, algumas limitações podem ser destacadas. Houve a derivação de respostas a partir de perguntas amplas, impossibilitando o desenvolvimento e aprofundamento de algumas análises. Apesar de respostas autorreferidas já estarem validadas para uso de estudos populacionais principalmente, outra limitação foi a obtenção da informação referida para as variáveis. Dados sobre comportamentos socialmente indesejáveis são propensos a subestimativas (ZAITUNE et al., 2012). Uma vez que se limitou a um delineamento de corte transversal, possibilitou o viés de causalidade reversa, não sendo possível identificar se as variáveis antecederam ou sucederam-se ao período de coleta de dados. Estudos futuros longitudinais são fortemente recomendados. É possível ter sido um estudo pouco representativo dos idosos com múltiplas incapacidades ou declínio cognitivo, sendo que tais indivíduos com a possibilidade de os mesmos terem maior número de comportamentos negativos relacionados à saúde foram excluídos inicialmente do estudo de acordo com a metodologia proposta. Por fim, investigar comportamentos relacionados à saúde pode gerar um efeito de se ater apenas aos determinantes individuais da vida, e desconsiderar os determinantes múltiplos da saúde. Ações, intervenções, fiscalização, projetos e programas governamentais, também das organizações privadas e da sociedade civil são necessários para complementar ações para esse fim (KOHL III et al., 2012).

\section{Conclusão}

Embora não tenham sido encontradas altas frequências de comportamentos negativos em saúde, o sedentarismo e a não busca por serviços odontológicos continuam sendo aspectos desafiadores entre idosos. Os dados, porém, contribuem com a literatura gerontológica ao indicar que os comportamentos relacionados à saúde de idosos devem ser considerados de forma multidimensional. Faz-se importante, em estudos futuros, tanto de caráter quantitativo como qualitativo, a exploração detalhada das outras características além das sociodemográficas que influenciam os mesmos, como arranjo familiar e ocupação. 
Esse estudo como referência pode estimular futuras pesquisas sobre a magnitude dos indicadores de saúde em idosos. Os fatores associados identificados podem contribuir para o planejamento de serviços de saúde, numa perspectiva amplificada, que incentivem e orientem os idosos quanto à importância do autocuidado. Sugere-se que os profissionais da saúde estejam atentos aos diferentes perfis de comportamentos relacionados à saúde aderidos pelos idosos, o que auxiliaria na prática clínica, e levaria a melhorias nos serviços de saúde e a políticas nacionais mais eficientes em relação à população idosa e ao envelhecimento.

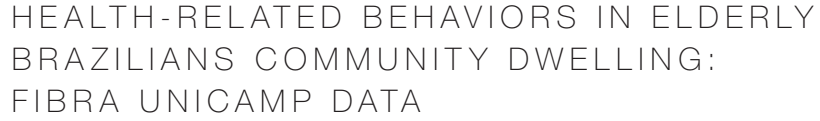

abstract

It attempted to describe and identify associations between healthrelated behaviors among themselves and with sociodemographic variables in the elderly community dwelling. The study, descriptive, population character, and cross-sectional analysis, is part of the electronic bank FIBRA Study - Unicamp pole -, with a random sample of 2552 older, with a mean age of 72.3 years $(\mathrm{SD}= \pm 5.5$ ) and consisting primarily of women (66\%). Multiple correspondence analysis showed associations between variables, pointing to the trend of formation of three behavioral sample profiles: 1) "Engaged in unhealthy behaviors", 2) "Engaged in Healthy Behaviors" and 3) "Indifferent or passive front the health". Ratifying the literature, the health-related behaviors of the elderly should be considered in a multidimensional way generating synergy between each other. Such information contributes to the design of strategies in health care and welfare for the elderly.

keywords

Healthy behaviors. Aging. Elderly. Health promotion.

referências

ALDWIN, Carolyn. M.; PARK, Crystal L.; SPIRO III, Avron (Ed.). Handbook of health psychology and aging. 1. ed. New York: The Guilford Press, 2007. 
BRASIL. Secretaria de Vigilância em Saúde. Vigitel Brasil 2013: vigilância de fatores de risco e proteção para doenças crônicas por inquérito telefônico. Brasília, DF: Ministério da Saúde, 2014. Disponível em: <http://bvsms.saude.gov.br/bvs/publicacoes/ vigitel_brasil_2013.pdf>. Acesso em: 9 nov. 2015.

Vigitel Brasil 2011: vigilância de fatores de risco e proteção para doenças crônicas por inquérito telefônico. Brasília, DF: Ministério da Saúde, 2012. Disponível em: <http://bvsms.saude.gov.br/bvs/publicacoes/vigitel_brasil_2011_fatores_risco_doencas_cronicas.pdf $>$. Acesso em: 9 nov. 2015

Ministério da Saúde. Fundação Nacional de Saúde. Informe técnico - Campanha Nacional de Vacinação do Idoso 2010. Brasília, DF: Ministério da Saúde, 2010. Disponível em: <http://cve.saude.sp.gov.br>. Acesso em: 9 nov. 2015.

. Fundação Nacional de Saúde. Informe técnico - Campanha Nacional de Vacinação do Idoso 2009. São Paulo: Ministério da Saúde, 2009. Disponível em: <ftp://ftp.cve.saude.sp.gov.br/doc_tec/imuni/if09_influ.pdf>. Acesso em: 9 nov. 2015.

CAMPOS, E. C. et al. Fatores relacionados à vacinação contra a gripe em idosos: estudo transversal, Cambé, Paraná, Brasil. Cadernos de Saúde Pública, Rio de Janeiro, v. 28, n. 5, p. 878-888, maio 2012.

COSTA, T. B.; NERI, A. L. Medidas de atividade física e fragilidade em idosos: dados do FIBRA Campinas, São Paulo, Brasil. Cadernos de Saúde Pública, Rio de Janeiro, v. 27, n. 8, p. 1537-1550, ago. 2011.

CRAMM, J. M. et al. The role of disease management programs in the health behavior of chronically ill patients. Patient Educational and Counselling, Limerick, v. 95, p. 137142, abr. 2014.

DAWSON, D. A. et al. Effectiveness of the derived alcohol use disorders identification test (AUDIT-C) in screening for alcohol use disorders and risk drinking in the US general population. Alcoholism, Clinical and Experimental Research, New York, v. 29, n. 5, p. 844-854, maio 2005.

DESCRITORES em Ciências da Saúde: DeCS. ed. rev. e ampl. São Paulo: BIREME; OPAS; OMS, 2015. Disponível em: <http://decs.bvsalud.org>. Acesso em: 9 nov. 2015.

DODD, L. et al. Lifestyle risk factors of students: a cluster analytical approach. Preventive Medicine, New York, v. 51, n. 1, p. 73-77, jul. 2010.

DUARTE, Y. A. O. et al. Impacto do sedentarismo na incidência de doenças crônicas e incapacidades e na ocorrência de óbitos entre os idosos do município de São Paulo. Saúde Coletiva, São Paulo, v. 5, n. 24, p. 183-188, jan. 2008.

EMILE, M. et al. Aging stereotypes and active lifestyle: personal correlates of stereotype internalization and relationships with level of physical activity among older adults. Psychology of Sports and Exercise, v. 15, n. 2, p. 198-204, mar. 2014.

FRANCISCO, P. M. S. B. et al. Vacinação contra influenza em idosos: prevalência, fatores associados e motivos de não-adesão em Campinas, São Paulo, Brasil. Cadernos de Saúde Pública, Rio de Janeiro, v. 27, n. 3, p. 417-26, mar. 2011.

HASKELL, W. et al. Physical activity and public health: updated recommendation for adults from the american college of sports medicine and the american heart association. Medicine \& Science in Sports \& Exercise, v. 39, n. 8, p. 1423-1434, ago. 2007.

INSTITUTO BRASILEIRO DE GEOGRAFIA E ESTATíSTICA (IBGE). Ministério do Planejamento, Orçamento e Gestão. Pesquisa Nacional de Saúde 2013: acesso e utilização dos serviços de saúde, acidentes e violências. Rio de Janeiro: IBGE, 2015. Disponível em: <http://biblioteca.ibge.gov.br/biblioteca-catalogo? $v i e w=$ detalhes\&id=294074> . Acesso em: 9 nov. 2015.

. Pesquisa Nacional de Saúde 2013: percepções do estado de saúde, estilos de vida e doenças crônicas. Rio de Janeiro: IBGE, 2014. Disponível em: < http:// 

nov. 2015

JOMAR, R. T.; ABREU, A. M.; GRIEP, R. H. Padrões de consumo de álcool e fatores associados entre adultos usuários de serviço de atenção básica do Rio de Janeiro, RJ, Brasil. Ciências \& Saúde Coletiva, Rio de Janeiro, v. 19, n. 1, p. 27-37, 2014.

KENDIG, H. et al. Health, lifestyle, and gender influences on aging well: an Australian longitudinal analysis to guide health promotion. Frontiers in Public Health, Lausana, v. 2, n. 70, p. 1-9, jul. 2014

$\mathrm{KOHL} I \mathrm{II}, \mathrm{H}$. et al. The pandemic of physical inactivity: global action for public health. The Lancet, London, v. 380, n. 21, p. 294-305, jul. 2012

LÄMMLE, L. et al. Does initial behavior predict our physical fitness and health 18 years later? Psychology of Sport and Exercise, v. 15, p. 81-88, jan. 2014

LEBRÃO, M. L.; LAURENTI, R. Saúde, bem-estar e envelhecimento: o Estudo SABE no município de São Paulo. Revista Brasileira de Epidemiologia, v. 8, n. 2, p. 127-141, jun. 2005

LIMA-COSTA, M. F. F. et al. Comportamentos em saúde entre idosos hipertensos, Brasil, 2006. Revista de Saúde Pública, São Paulo, v. 43, n. 2, p. 18-26, 2009.

LOUVISON, M. C. P. et al. Desigualdades no uso e acesso aos serviços de saúde entre idosos do município de São Paulo. Revista de Saúde Pública, São Paulo, v. 42, n. 4, p. 733-740, ago. 2008.

MADRUGA, C. et al. Tobacco use among the elderly: the first Brazilian National Survey (BNAS). Aging \& Mental Health, v. 14, n. 6, p. 720-724, jun. 2010.

MIOTTO, M. H. M. B.; BARCELLOS, L. A.; VELTEN, D. B. Avaliação do impacto na qualidade de vida causado por problemas bucais na população adulta e idosa em município da região sudeste. Ciência \& Saúde Coletiva, Rio de Janeiro, v. 17, n. 2, p. 397-406, 2012.

MOURA, R. F. Fatores associados à vacinação anti-influenza em idosos: um estudo baseado na pesquisa Saúde, Bem-Estar e Envelhecimento - SABE. 2013. 102 f. Dissertação (Mestrado em Ciências)-Universidade de São Paulo, São Paulo, 2013.

NELSON, M. et al. Physical activity and public health in older adults: recommendation from the American College of Sports Medicine and the American Heart Association. Circulation, v. 116, n. 9, p. 1094-1105, aug. 2006

NERI, A. L. O legado de Paul B. Baltes à psicologia do desenvolvimento e do envelhecimento. Temas em Psicologia, Florianópolis, v. 14, n 1, p. 17-34, 2007.

NERI, A. L. et al. Metodologia e perfil sociodemográfico, cognitivo e de fragilidade de idosos comunitários de sete cidades brasileiras: estudo FIBRA. Caderno de Saúde Pública, Rio de Janeiro, v. 29, n. 4, p. 778-792, abr. 2013.

ORGANIZAÇÃO MUNDIAL DA SAÚDE (OMS). Global status report on alcohol and health. WHO: Luxemburgo, 2014. Disponivel em: <http://who.int/substance_abuse/ publications/global_alcohol_report/en/>. Acesso em: 9 nov. 2015.

PALACIOS-CENÃ, D. et al. Has the prevalence of health care services use increased over the last decade (2001-2009) in elderly people?: a Spanish population-based survey. Maturitas, Limerick, v. 76, n. 4, p. 326-333, dez. 2013.

PEREIRA, G. N. et al. Indicadores demográficos e socioeconômicos associados à incapacidade funcional em idosos. Caderno de Saúde Pública, Rio de Janeiro, v. 28, n. 11, p. 2035-2042, nov. 2012.

PRUCHNO, R.; WILSON-GENDERSON, M. Adherence to clusters of health behaviors and successful aging. Journal of Aging and Health, v. 24, n. 8, p. 1279-1297, set. 2012. 
SENGER, A. E. V. et al. Alcoolismo e tabagismo em idosos: relação com ingestão alimentar e aspectos socioeconômicos. Revista Brasileira de Geriatria e Gerontologia, Rio de Janeiro, v. 14, n. 4, p. 713-719, 2011.

SMITH, P.; GLAZIER, R. H.; SIBLEY, L. M. The predictors of self-rated health and the relationship between self-rated health and health service needs are similar across socioeconomic groups in Canada. Journal of Clinical Epidemiology, Oxford, v. 63, n. 4, p. 412-421, abr. 2010

TEJADA, C. A. O. et al. Factors associated with smoking cessation in Brazil. Cadernos de Saúde Pública, Rio de Janeiro, v. 29, n. 8, p. 1555-64, ago. 2013.

VAGETTI, G. C. et al. The prevalence and correlates of meeting the current physical activity for health guidelines in older people: a cross-sectional study in Brazilian women. Archives of Gerontology and Geriatrics, v. 56, n. 3, p. 492-500, maio/jun. 2013.

VARGAS, D.; BITTENCOURT, M. N.; BARROSO, L. P. Padrões de consumo de álcool de usuários de serviços de atenção primária à saúde de um município brasileiro. Ciências \& Saúde Coletiva, Rio de Janeiro, v. 19, n. 1, p. 17-25, 2014.

VIANA, D. A,; RODRIGUES, L. R.; TAVARES, D. M. Fatores sociodemográficos e econômicos associados ao tabagismo na população idosa. Jornal Brasileiro de Psiquiatria, Rio de Janeiro, v. 63, n. 3, p. 220-6, set. 2014.

VRDOLJAK, D. et al. Lifestyle intervention in general practice for physical activity, smoking, alcohol consumption and diet in elderly: a randomized controlled trial. Archives of Gerontology and Geriatrics, Amsterdã, v. 58, n. 1, p. 160-169, jan./fev. 2014

WHITLEY, E. et al. The role of health behaviours across the life course in the socioeconomic patterning of all-cause mortality: the West of Scotland 27-prospective cohort study. Annals of Behavioral Medicine, Knoxville, v. 47 n. 2, p. 148-157, abr. 2014.

ZAITUNE, M. P. A. et al. Fatores associados ao tabagismo em idosos: inquérito de saúde no estado de São Paulo (ISA-SP). Cadernos de Saúde Pública, Rio de Janeiro, v. 28, n. 3, p. 583-595, mar. 2012 
